01,09

\title{
Электронная структура и оптические свойства соединения $\mathrm{FeAl}_{2}$
}

\author{
(C) Ю.В. Князев ${ }^{1}$, А.В. Лукоянов ${ }^{1,2}$, Ю.И. Кузьмин ${ }^{1}$ \\ ${ }^{1}$ Институт фризики металлов им. М.Н. Михеева УрО РАН, \\ Екатеринбург, Россия \\ ${ }^{2}$ Уральский федеральный университет им. Б.Н. Ельцина, \\ Екатеринбург, Россия \\ E-mail: knyazev@imp.uran.ru
}

Поступила в Редакцию 16 сентября 2019 г.

В окончательной редакции 16 сентября 2019 г.

Принята к публикации 16 сентября 2019 г.

Проведены исследования электронной структуры и оптических свойств бинарного интерметаллического соединения $\mathrm{FeAl}_{2}$. Выполнены спин-поляризованные расчеты электронной структуры, определены магнитные моменты атомов. С использованием эллипсометрического метода в спектральном интервале $0.22-15 \mu \mathrm{m}$ измерены оптические характеристики материала. Показано, что экспериментальная межзонная оптическая проводимость соединения удовлетворительно интерпретируется на основе рассчитанной плотности электронных состояний.

Ключевые слова: соединение $\mathrm{FeAl}_{2}$, электронная структура, оптические свойства.

DOI: 10.21883/FTT.2020.01.48739.580

\section{1. Введение}

Согласно диаграмме состояний системы железо-алюминий [1] в сплавах данного типа образуется шесть стабильных кристаллических фаз, каждая из которых обладает большим разнообразием физико-химических свойств. Проводимые в последние годы исследования структурных, механических, магнитных, тепловых, электронных и др. характеристик показали, что соединения системы $\mathrm{Fe}-\mathrm{Al}$, помимо фундаментального изучения, заслуживают внимание как объекты, перспективные для практического применения. В частности, благодаря высокой стойкости к окислению, они являются подходящими кандидатами для использования при высоких температурах. Одним из материалов, принадлежащих к данному семейству, является бинарное соединение $\mathrm{FeAl}_{2}$ с триклинной кристаллической решеткой, обладающее целым рядом интересных особенностей электронных и магнитных свойств. Исследования [2,3] показали, что в широком температурном интервале электросопротивление этого соединения почти не зависит от температуры, что, как правило, свойственно полуметаллам. При $T_{\mathrm{N}} \approx 47 \mathrm{~K}$ соединение переходит в антиферромагнитную фазу [4,5], характеризуемую антипараллельным упорядочением магнитных моментов железа. При температурах ниже $T_{\mathrm{S}} \approx 32 \mathrm{~K}$ наблюдается состояние спинового стекла, о чем свидетельствуют температурные зависимости магнитной восприимчивости [3,6,7]. Показано $[6,8]$, что замещение алюминия атомами другого $p$-металла существенно влияет на величины указанных температур, при этом $T_{\mathrm{N}}$ растет, а $T_{\mathrm{S}}$ понижается. Изучение тепловых свойств исследуемого интерметал- лида $[2,9,10]$ указывают на возможность его использования в качестве перспективного термоэлектрического материала.

Разнообразие физических свойств $\mathrm{FeAl}_{2}$ стимулирует интерес к исследованию его электронной структуры. Для данного соединения рядом авторов [11-13] были выполнены расчеты энергетического спектра $E(k)$, в частности, определена природа различных электронных состояний вблизи уровня Ферми $E_{\mathrm{F}}$. Следует отметить, что результаты указанных вычислений существенно отличаются друг от друга, что вызывает необходимость проведения дальнейших исследований. Кроме того, расчеты $E(k)$ проводились не только для триклинной кристаллической структуры, но также для гипотетической тетрагональной фазы типа $\mathrm{MoSi}_{2}$, в которой прогнозируется существование узкой $(\sim 0.1-0.2 \mathrm{eV})$ энергетической щели, локализованной непосредственно на $E_{\mathrm{F}}$ [14]. Высокотемпературный синтез такой фазы возможен при давлении $\sim 10 \mathrm{GPa}$. В настоящей работе для получения информации об электронной структуре соединения $\mathrm{FeAl}_{2}$ используется метод, сочетающий первопринципные спин-поляризованные расчеты зонной структуры и экспериментальные исследования оптических свойств, впервые выполненные для данного материала.

\section{2. Расчет электронной структуры и эксперимент}

Соединение $\mathrm{FeAl}_{2}$ имеет триклинную элементарную ячейку с параметрами кристаллической структуры: $a=4.878 \AA, b=6.461 \AA$ и $c=8.800 \AA ; \alpha=91.75^{\circ}$, $\beta=73.27^{\circ}$ и $\gamma=96.89^{\circ}$ (пространственная группа $P 1$ ). 
Ионы железа и алюминия в такой структуре занимают 18 позиций, включающих 10 позиций Al, 5 позиций $\mathrm{Fe}$ и 3 позиции со смешанным заполнением $\mathrm{Al} / \mathrm{Fe}$ (обозначаемые как M1, M2, M3), с одной точечной группой симметрии $1 a(x, y, z)$, но различными значениями координат. Для расчетов электронной структуры использовались позиции, идентифицированные в экспериментальной работе [15]. В указанной кристаллической структуре $\mathrm{FeAl}_{2}$ каждый ион $\mathrm{Fe}$ находится в окружении из 10-11 ближайших соседей, по большей части ионов $\mathrm{Al}$, а также в среднем 1.6 ионов $\mathrm{Fe}$ и 1.8 узлов со смешанным заполнением Al/Fe [3].

Электронная структура соединения $\mathrm{FeAl}_{2}$ была рассчитана в пакете программ Quantum ESPRESSO [16] c использованием обменно-корреляционного потенциала в приближении обобщенной градиентной поправки (GGA) [17] версии Perdew-Burke-Ernzerhof [18]. В pacчетах использовались псевдопотенциалы из стандартной библиотеки Quantum ESPRESSO [19]. Интегрирование в обратном пространстве производилось по сетке из $6 \times 4 \times 2=48$ k-точек. Волновые функции в расчете раскладывались по плоским волнам, в цикле самосогласования для достижения сходимости использовался энергетический предел для плоских волн, равный 60 Ry.

В расчетах, в соответствие с экспериментальными данными [3], моделировалось антиферромагнитное расположение магнитных моментов ионов железа. Ионы алюминия в расчете остаются практически немагнитными с максимальным значением $0.02 \mu_{\mathrm{B}}$. Для ионов железа полученные в результате самосогласованных расчетов значения спиновой поляризации соответствуют полному магнитному моменту $0.48 \mu_{\mathrm{B}}$ на одну формульную единицу $\mathrm{FeAl}_{2}$. При этом на различных ионах железа в зависимости от окружения магнитный момент составляет от 0.8 до $2.1 \mu_{\mathrm{B}}$. Следует отметить, что, согласно магнитным измерениям для $\mathrm{FeAl}_{2}$, экспериментальные оценки эффективного магнитного момента на ион железа из закона Кюри-Вейсса составляют $1.32 \mu_{\mathrm{B}}$ [20] и $2.55 \mu_{\mathrm{B}}$ [3]. Для близких по составу сплавов с небольшим избыточным содержанием алюминия $\mathrm{Fe}_{2} \mathrm{Al}_{5}$ и $\mathrm{Fe}_{4} \mathrm{Al}_{13}$ магнитные моменты равны 0.73 и $0.44 \mu_{\mathrm{B}}$, соответственно [21]. Теоретические величины магнитных моментов находятся в хорошем согласии с экспериментальными оценками.

На рис. 1 представлена полная плотность электронных состояний $N(E)$ для двух направлений спина $\uparrow$ и $\downarrow$. Здесь же приводятся парциальные плотности состояний для $3 d-, 4 p$ - и $4 s$-электронов $\mathrm{Fe}$ и $3 p$ - и $3 s$-электронов $\mathrm{Al}$. Расчет показал, что для рассматриваемых кристаллографических позиций $3 d$-зоны железа локализованы в интервале $-3<E_{\mathrm{F}}<2 \mathrm{eV}$. Вклады в $N(E)$ от других парциальных плотностей $\mathrm{Fe}$ и $\mathrm{Al}$, как показывает рис. $1, b, c$, существенно более протяженны и формируют энергетические полосы, которые по своей структуре идентичны для обеих спиновых проекций.

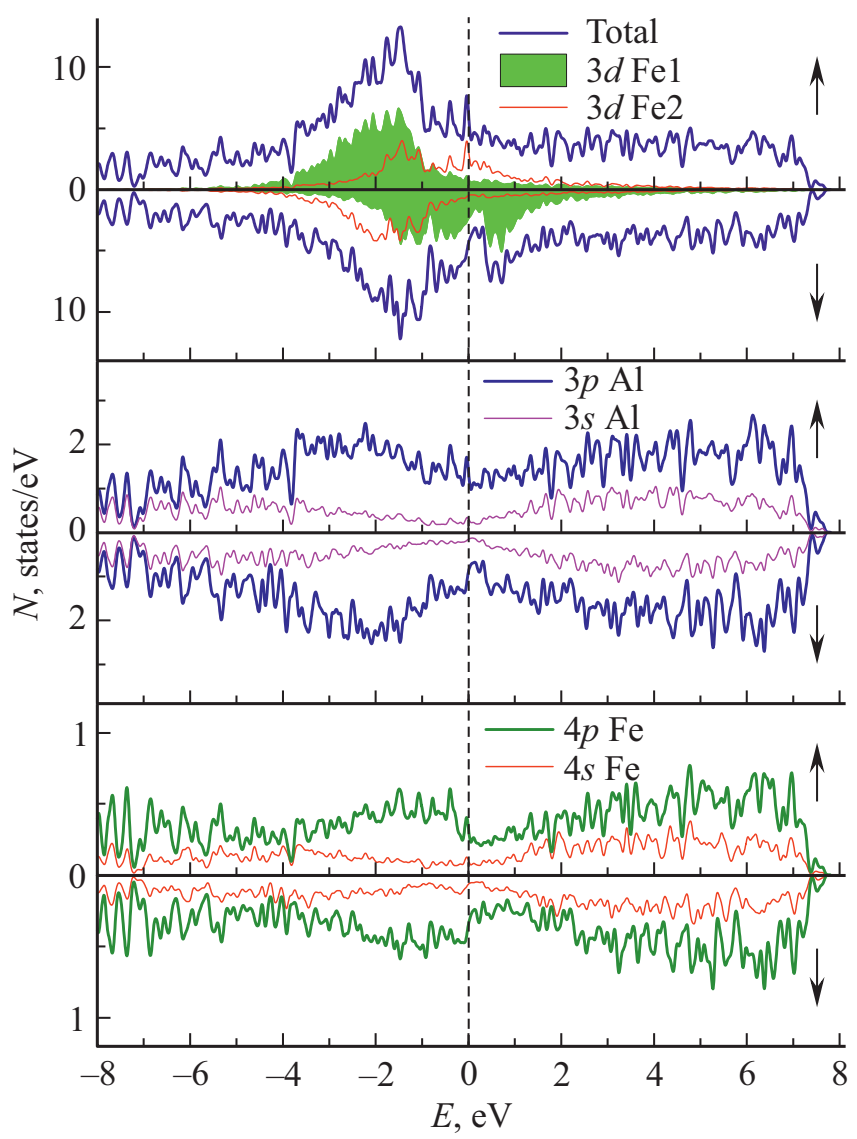

Рис. 1. Полная и парциальные (для $3 d-, 4 p$-, $4 s$-электронов $\mathrm{Fe}$ и $3 p$-, $3 s$-электронов $\mathrm{Al}$ ) плотности электронных состояний соединения $\mathrm{FeAl}_{2}$

Поликристаллический образец соединения $\mathrm{FeAl}_{2}$ был приготовлен по методу [2] в индукционной печи при сплавлении составных компонентов в атмосфере чистого аргона с последующим многочасовым отжигом в вакууме при $850^{\circ} \mathrm{C}$. Результаты рентгеноструктурного анализа, проведенного на дифрактометре ДРОН-6 в $\mathrm{Cu} K_{\alpha}$ излучении, подтвердили однофазность триклинной структуры с параметрами кристаллической решетки, близкими к полученным в [15]. Оптические свойства соединения исследованы при комнатной температуре в интервале длин волн $\lambda=0.22-15 \mu \mathrm{m}(E=0.083-5.64 \mathrm{eV})$ эллипсометрическим методом Битти, основанным на изменении поляризации света при отражении от поверхности образца с определением отношения амплитуд и разности фаз света $s$ - и $p$-поляризаций. Зеркальная поверхность образца была получена механическим полированием на алмазных пастах различной зернистости. По экспериментальным значениям оптических постоянных (показателей преломления $n(\lambda)$ и поглощения $k(\lambda))$ рассчитан ряд спектральных параметров соединения: диэлектрическая проницаемость, оптическая проводимость и отражательная способность. 


\section{3. Результаты и обсуждение}

Зависимости $n$ и $k$ от длины волны падающего света приведены на рис. 2. Во всем спектральном диапазоне выполняется неравенство $k>n$, характерное для материалов с металлическим типом проводимости. При этом действительная часть диэлектрической проницаемости $\varepsilon_{1}=n^{2}-k^{2}$ является отрицательной величиной (рис. 3), что также типично для подобных систем. На вставке рис. 3 приводится энергетическая зависимость отражательной способности $R=\left[(n-1)^{2}+k^{2}\right] /\left[(n+1)^{2}+k^{2}\right]$ соединения. На всем протяжении исследуемого спектрального интервала величина $R$ растет с уменьше-

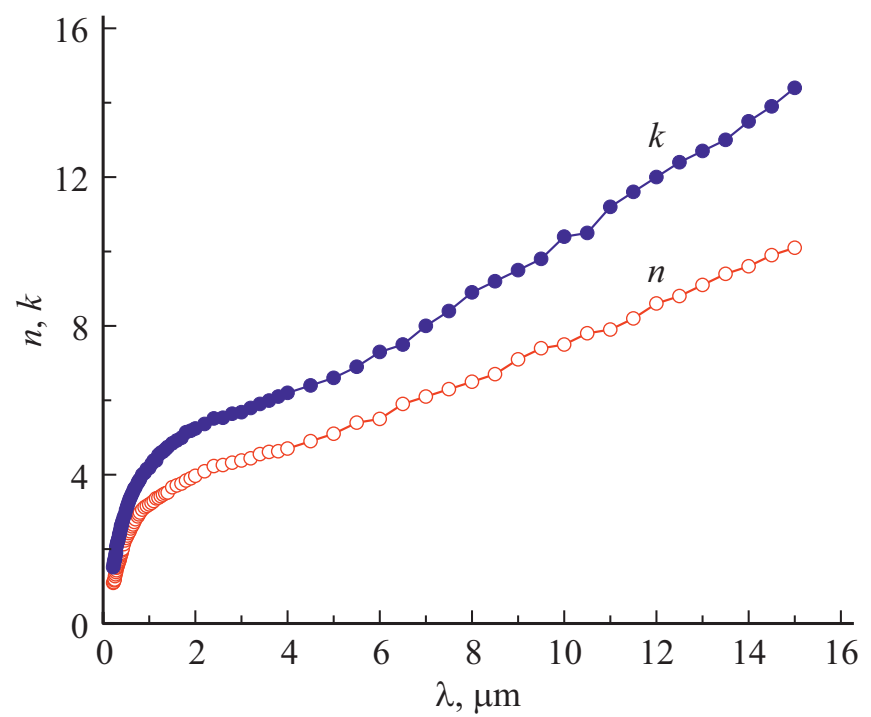

Pис. 2. Дисперсия оптических постоянных $n$ и $k$ соединения $\mathrm{FeAl}_{2}$.

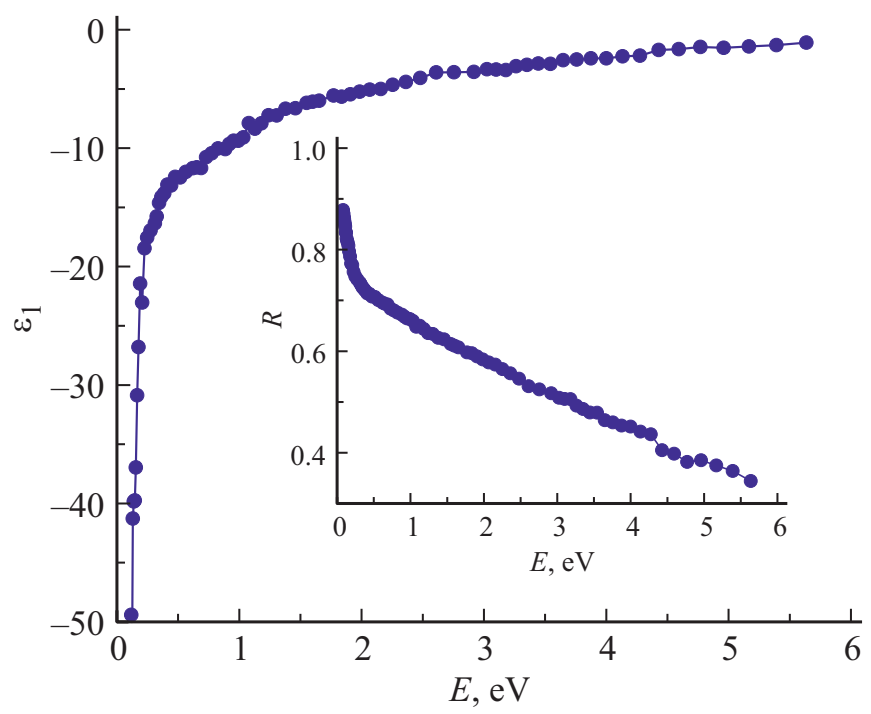

Pис. 3. Действительная часть диэлектрической проницаемости $\varepsilon_{1}$ и отражательная способность $R$ (вставка) соединения $\mathrm{FeAl}_{2}$.

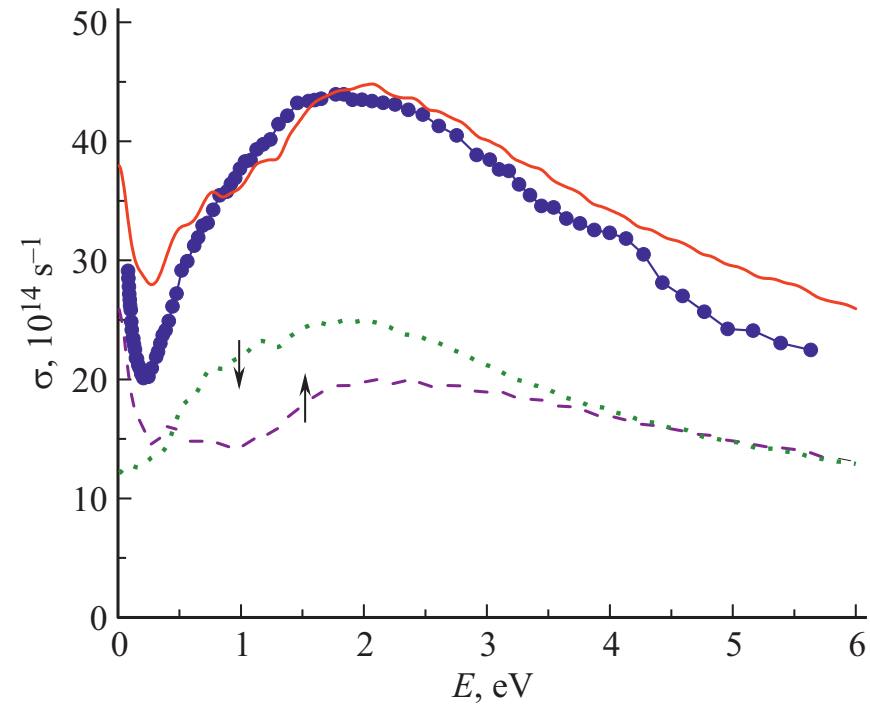

Рис. 4. Энергетическая зависимость экспериментальной и теоретической оптической проводимости соединения $\mathrm{FeAl}_{2}$. Показаны также вклады от двух спиновых подсистем ( $\uparrow$ и $\downarrow)$.

нием энергии кванта, приближаясь к значению $\sim 0.9$, что несколько ниже, чем для высокопроводящих материалов, где при соответствующих энергиях величина отражательной способности достигает почти единицы. Экспериментальный спектр оптической проводимости $\sigma(E)=n k \omega / 2 \pi \quad(\omega-$ частота света $)$, характеризующей частотную дисперсию и интенсивность оптического отклика среды, представлен на рис. 4. В отличие от статической проводимости, данный параметр зависит не только от плотности электронных состояний на уровне Ферми, но также учитывает структуру $N(E)$ во всем исследуемом диапазоне частот. Форма приведенной на рисунке кривой указывает на то, что ниже энергии $\sim 0.2 \mathrm{eV}$, где наблюдается ярко выраженный минимум, дисперсия $\sigma(E)$ определяется внутризонным (друдевским) механизмом возбуждения электронов квантами света малых энергий $\left(\sigma \sim 1 / \omega^{2}\right)$. На данном участке спектра по оптическим постоянным могут быть рассчитаны такие параметры электронов проводимости, как концентрация $N$, плазменная $\omega_{\mathrm{p}}$ и релаксационная $\gamma$ частоты. Величина плазменной частоты, характеризующей коллективные осцилляции свободных электронов, принимает значение $\omega_{\mathrm{p}}=3.9 \cdot 10^{15} \mathrm{~s}^{-1}$. При этом частота релаксации, аддитивно учитывающая все типы рассеяния электронов при их возбуждении электромагнитной волной, равна $\gamma=3.5 \cdot 10^{14} \mathrm{~s}^{-1}$. Концентрация электронов проводимости составляет $N=4.3 \cdot 10^{21} \mathrm{~cm}^{-3}$, что на порядок меньше, чем в нормальных металлах.

При энергиях выше $\sim 0.2 \mathrm{eV}$ характер зависимости $\sigma(E)$ свидетельствует о доминирующей роли межзонного поглощения света. В этом интервале спектра расположена почти симметричная по форме широкая 
абсорбционная полоса с максимумом вблизи $2 \mathrm{eV}$. Образование данной полосы связано с квантовыми переходами между электронными состояниями ниже и выше $E_{\mathrm{F}}$ и определяется зонной структурой соединения, что позволяет объяснить природу ее появления на основе приведенного на рис. 1 расчета плотности состояний. С этой целью, в соответствии с методом [22] на основе свертки $N(E)$ в энергетическом диапазоне $E_{\mathrm{F}} \pm 6 \mathrm{eV}$ был вычислен суммарный вклад всех типов межзонных переходов в оптическую проводимость при условии их равной вероятности с учетом аддитивности вкладов от $\uparrow$ и $\downarrow$ электронных зон. Результат такого расчета приведен на рис. 4 совместно с экспериментальным спектром $\sigma(E)$. Отметим, что данный расчет носит качественный характер, поскольку не учитывает вероятности квантовых переходов. Тем не менее, сравнение показывает наличие существенного сходства экспериментальной и теоретической зависимостей. В частности, расчет воспроизводит наблюдаемую картину межзонного поглощения, предсказывая широкую полосу практически идентичной формы с максимумом, близким по энергии к эмпирически полученному значению. В формирование данной полосы, как следует из структуры спектра $N(E)$, основной вклад вносят межзонные переходы с участием $3 d$-состояний атомов железа, локализованных в обеих неэквивалентных позициях. Большая ширина $d$-зоны и существенная $s$-, $p$-, $d$-гибридизация электронных состояний атомов $\mathrm{Fe}$ и Al обусловливают интенсивные электронные переходы в широкой области энергий, что подтверждается видом спектра оптической проводимости. Другие типы переходов, связанные только с $s$ - и $p$-зонами, ввиду низких значений их парциальных плотностей состояний, дают в оптическую проводимость вклады, существенно меньшие по величине. На рис. 4 также представлены расчетные кривые $\sigma_{\uparrow}(E)$ и $\sigma_{\downarrow}(E)$ для электронных зон с различными спиновыми проекциями, указывающие на то, что межзонные переходы реализуются в обеих подсистемах. Обращает внимание определенное различие в поведении этих зависимостей: если спектр $\sigma_{\downarrow}(E)$ имеет сходство со спектром суммарной кривой $\sigma(E)$, то форма $\sigma_{\uparrow}(E)$ существенно отличается. В структуре данной составляющей наблюдается два максимума (вблизи 0.4 и $2.0 \mathrm{eV}$ ), а также резкий подъем в области низких частот, соответствующей ИК области. Расчет показывает, что в обеих системах зон межзонные переходы могут начинаться практически с нулевой энергии, а их интенсивность на протяжении почти всего диапазона длин волн (за исключением низкоэнергетической части $E<\sim 0.2 \mathrm{eV}$ ) сопоставима по величине. В целом, можно констатировать, что проведенные оптические измерения и результаты первопринципных расчетов дают взаимно согласованное описание электронной структуры соединения $\mathrm{FeAl}_{2}$.

\section{4. Заключение}

Представлены результаты исследования электронной структуры и спектральных свойств бинарного соединения $\mathrm{FeAl}_{2}$. Методом эллипсометрии в широком диапазоне спектра измерены оптические характеристики, определены плазменные и релаксационные частоты электронов проводимости. В рамках самосогласованного $a b$ initio расчета получена информация о спинполяризованных плотностях электронных состояний интерметаллида в интервале $E_{\mathrm{F}} \pm 8 \mathrm{eV}$, которая используется для интерпретации экспериментальных результатов. Показано, что характер частотной дисперсии оптической проводимости в области основной полосы межзонного поглощения и основные структурные особенности удовлетворительно описываются на основе проведенного расчета указанной функции.

\section{Финансирование работы}

Работа выполнена в рамках государственного задания Минобрнауки России (тема „Электрон“, № AАAА-A18118020190098-5).

\section{Конфликт интересов}

Авторы зявляют, что у нит нет конфликта интересов.

\section{Список литературы}

[1] B. Sundman, I. Ohnuma, N. Dupin, U.R. Kattner, S.G. Fries. Acta Mater. 57, 2896 (2009).

[2] C.S. Lue, Y-K. Kuo. J. Phys.: Condens. Matter 15, 877 (2003).

[3] S.C. Lue, Y. Öner, D.G. Naugle, J.H. Ross, Jr. Phys. Rev. B 63, 184405 (2001).

[4] T. Ghosh, T. Fukuda, T. Kakeshita, S.N. Kaul, P.K. Mukhopadhyay. J. Alloys Compd. 782, 915 (2019).

[5] D. Kaptás, E. Sváb, Z. Somogyvári, G. André, L.F. Kiss, J. Balogh, L. Bujdosó, T. Kemény, I. Vincze. Phys. Rev. B 73, 012401 (2006).

[6] Z. Jagličić, S. Vrtnik, M. Feuerbacher, J. Dolinšek. Phys. Rev. B 83, 224427 (2011).

[7] J. Chi, Y. Li, F.G. Vagizov, V. Goruganti, J.H. Ross, Jr. Phys. Rev. B 71, 024431 (2005).

[8] T. Ghosh, T. Fukuda, T. Kakeshita, S.N. Kaul, P.K. Mukhopadhyay. Phys. Rev. B 95, 140401 (2017).

[9] K. Tobita, N. Sato, K. Kitahara, Y. Takagiwa, K. Kimura. Mater. Trans. 57, N 7, 1045 (2016).

[10] T. Zienert, A. Leineweber, O. Fabrichnaya. J. Alloys. Compd. 725, 848 (2017).

[11] M. Weinert, R.E. Watson. Phys. Rev. B 58, 9732 (1998).

[12] J. Pan, J. Ni, B. Yang. Comput. Mater. Sci. 50, 2433 (2011).

[13] Y. Liu, X. Chong, Y. Jiang, R. Zhou, J. Feng. Physica B: Condens. Mater. 506, 1 (2017).

[14] K. Tobita, N. Sato, Y. Katsura, K. Kitahara, D. Nishio-Hamane, H. Gotou, K. Kimura. Scripta Mater. 141, 107 (2017).

[15] M. Khaidar, C.H. Allibert, J. Driole. Z. Metallkunde 73, 7, 433 (1982) 
[16] P. Giannozzi, O. Andreussi, T. Brumme, O. Bunau, M.B. Nardelli, M. Calandra, R. Car, C. Cavazzoni, D. Ceresoli, M. Cococcioni, N. Colonna, I. Carnimeo, A. Dal Corso, S. de Gironcoli, P. Delugas, R.A. DiStasio Jr., A. Ferretti, A. Floris, G. Fratesi, G. Fugallo, R. Gebauer, U. Gerstmann, F. Giustino, T. Gorni, J. Jia, M. Kawamura, H.-Y. Ko, A. Kokalj, E. Küçükbenli, M. Lazzeri, M. Marsili, N. Marzari, F. Mauri, N.L. Nguyen, H.-V. Nguyen, A. Otero-de-la-Roza, L. Paulatto, S. Poncé, D. Rocca, R. Sabatini, B. Santra, M. Schlipf, A.P. Seitsonen, A. Smogunov, I. Timrov, T. Thonhauser, P. Umari, N. Vast, X. Wu, S. Baroni. J. Phys.: Condens. Matter. 29, 465901 (2017).

[17] R.O. Jones. Rev. Mod. Phys. 87, 897 (2015).

[18] J.P. Perdew, K. Burke, M. Ernzerhof. Phys. Rev. Lett. 77, 3865 (1996).

[19] Fe.pbe-sp-van.UPF, Al.pbe-n-van.UPF. (Электронный pecypc) https://www.quantum-espresso.org/pseudopotentials.

[20] M.A. Taylor. Proc. Phys. Soc. London. 78, 1244 (1961).

[21] F. Müller, M. Rosenberg, W. Liu, U. Köster. Mater. Sci. Eng. A 134, 900 (1991)

[22] C.N. Berglund, W.E. Spicer. Phys. Rev. 136, A1044 (1964).

Редактор К.В. Емцев 